\title{
Paravalvular leak after transcatheter aortic valve implantation (TAVI): Short-term results. Data from Polish national POL-TAVI registry
}

\author{
Paweł Kleczyński ${ }^{1}$, Wojciech Zasada ${ }^{1}$, Maciej Bagieński ${ }^{1}$, Łukasz Rzeszutko ${ }^{1}$, \\ Danuta Sorysz ${ }^{1}$, Robert Sobczyński ${ }^{1}$, Jarosław Trębacz ${ }^{1}$, Bogdan Kapelak ${ }^{1}$, \\ Marian Zembala ${ }^{2}$, Dariusz Dudek ${ }^{1}$ \\ ${ }^{1}$ Institute of Cardiology, Jagiellonian University, Krakow, Poland \\ ${ }^{2}$ Silesian Center for Heart Diseases, Zabrze, Poland
}

\begin{abstract}
Background: The authors analyzed data from Polish national POL-TAVI registry in terms of paravalvular leak (PVL) occurrence after transcatheter aortic valve implantation (TAVI) and its impact on clinical outcomes.

Methods: A total of 331 patients with severe aortic stenosis underwent TAVI (Edwards Sapien, $n=141$; CoreValve, $n=190$ ). The grade of PVL and survival rate were assessed at 6-month follow-up.

Results: One hundred and eighty-one (54.7\%) patients developed mild PVL, and 22 (6.6\%) - moderate PVL after TAVI. No severe PVLs were observed. Occurrence of moderate PVL was device dependent and more frequent in the CoreValve group $(p=0.02)$. Larger CoreValve device used correlated with the grade of PVL (Spearman: $\rho=0.19 ; p=0.01$ ), but for Edwards Sapien devices this correlation was inverted (Spearman: $\rho=-0.62 ; p=0.01$ ). Six-month mortality rate was not significant between patients with no or mild PVL compared to those with moderate PVL $(p=0.12)$.

Conclusions: The PVL occurrence remains a frequent problem after TAVI and is device dependent, more common with Edwards $23 \mathrm{~mm}$ valve and $31 \mathrm{~mm}$ CoreValve prosthesis. (Cardiol J 2016; 23, 2: 163-168)
\end{abstract}

Key words: aortic stenosis, high-risk patients, TAVI, paravalvular leak

\section{Introduction}

Transcatheter aortic valve implantation (TAVI) is a less invasive treatment option for elderly, high-risk patients with symptomatic severe aortic stenosis (AS) than surgical aortic valve replacement (AVR); it improves survival [1] and quality of life [2] compared with medical treatment in inoperable patients. However, post-implantation paravalvular leak (PVL) remains a significant complication associated with TAVI, which may cause a higher mortality rates afterwards [3]. We sought to investigate the early- and mediumterm outcomes of TAVI in patients with PVL. Data from the Polish national POL-TAVI registry were analyzed.

Address for correspondence: Paweł Kleczyński, MD, PhD, Institute of Cardiology, Jagiellonian University, ul. Kopernika 17, 31-501 Kraków, Poland, e-mail: kleczu@interia.pl 


\section{Methods}

The POL-TAVI registry is an obligatory Polish registry for TAVI procedures in Poland. According to data reported by the registry, there were 381 TAVI implantations performed in Poland in 2013. Data of 331 (86.9\%) patients, for whom data regarding device type and PVL assessment were collected in POL-TAVI registry, were assessed. Fifty patients withdrew their consent for data analysis or data were incomplete for assessment. All patients who were meant to undergo TAVI were evaluated by a dedicated multidisciplinary Heart Team $[4,5]$. Clinical decision-making was based on a multimodality screening assessment including evaluation of surgical risk by logistic EuroScore I or Society of Thoracic Surgeons score. Additional risk criteria included porcelain aorta, advanced liver cirrhosis, severe neurological impairment, and frailty due to physician's judgment. Aortic annulus diameter was assessed with transesophageal echocardiography and computed tomography (CT) in each case. All procedures were performed by a dedicated hybrid team. Only Edwards Sapien, Ewards Sapien XT (Edwards Sapien, Irvine, United States) and Medtronic CoreValve (Medtronic CoreValve, Santa Rosa, United States) devices were used in this subset of patients. PVL at hospital discharge was graded as absent or mild, moderate and severe, according to the Valve Academic Research Consortium-2 (VARC 2) criteria with the use of transthoracic echocardiography [6]. We divided patients according to PVL degree into group PVL no higher than mild (no + mild), and at least moderate (moderate + severe).

\section{Definitions}

Cathlab is an examination room in a hospital with a single X-ray generator source and an image intensifier used to visualize the coronary arteries and treatment.

Hybrid operating room is a surgical theater that is equipped with advanced medical imaging devices such as fixed C-Arms, CT scanners enabling minimally invasive surgery or other procedures.

Operating room - operating theater for cardiosurgeons equipped with a mobile $\mathrm{C}$-arm.

\section{Statistical analysis}

All statistical analyses were performed using JMP Software (version 9.0.0; SAS Institute, Cary, NC, USA). Results were presented as numbers of patients (percentages) or median (interquartile
Table 1. Baseline demographic and clinical characteristic of enrolled patients.

\begin{tabular}{lc}
\hline Variable & $\mathrm{N}=331$ \\
\hline Age [years] & $80(75-84)$ \\
Female & $56 \%$ \\
Body mass index $\left[\mathrm{kg} / \mathrm{m}^{2}\right]$ & $26.8(23.9-29.7)$ \\
Arterial hypertension & $86 \%$ \\
Diabetes mellitus & $36 \%$ \\
Previous myocardial infarction & $26 \%$ \\
Previous CABG & $9.7 \%$ \\
Atrial fibrillation & $36 \%$ \\
Previous pacemaker & $14 \%$ \\
COPD & $16 \%$ \\
Chronic renal failure & $63 \%$ \\
Dialysis & $2.4 \%$ \\
CCS I-II & $41 \%$ \\
CCS III-IV & $59 \%$ \\
NYHA I-II & $23.5 \%$ \\
NYHA III-IV & $76.5 \%$ \\
Logistic EuroScore I [\%] & $15.7(9.5-24.8)$ \\
STS score [\%] & $5.3(3.1-11.5)$ \\
Aortic valve parameters: & \\
Pressure gradient max [mm Hg] & $80(65-98)$ \\
Pressure gradient mean [mm Hg] & $50(40-63)$ \\
Aortic valve area [cm $\left.{ }^{2}\right]$ & $0.7(0.6-0.8)$ \\
Left ventricular ejection fraction [\%] & $55(45-60)$ \\
\hline & \\
\hline
\end{tabular}

Data are presented as median values and interquartile range or numbers (percentages); CABG - coronary artery bypass grafting; COPD - chronic obstructive pulmonary disease; CCS - Canadian Cardiovascular Society scale; NYHA - New York Heart Association class; STS - Society of Thoracic Surgeons

range $[\mathrm{IQR}]$ ) where applicable. Correlation between PVL and other evaluated parameters were tested using $\chi^{2}$ or Pearson test, when applicable. Place of treatment and atrial fibrillation (AF) variables were introduced into nominal logistic model to assess odds ratio (OR) for each parameters included into model. All tests were two-tailed, and a $\mathrm{p}$ value $<0.05$ was considered statistically significant.

\section{Results}

POL-TAVI registry collected the data regarding aortic valve device which was implanted and assessment of post-procedural PVL of 331 patients. Baseline patient demographic, clinical and echocardiographic variables are presented in Table 1. Median of patients' age was 80 years and 56\% of patients were female. All patients had severe 


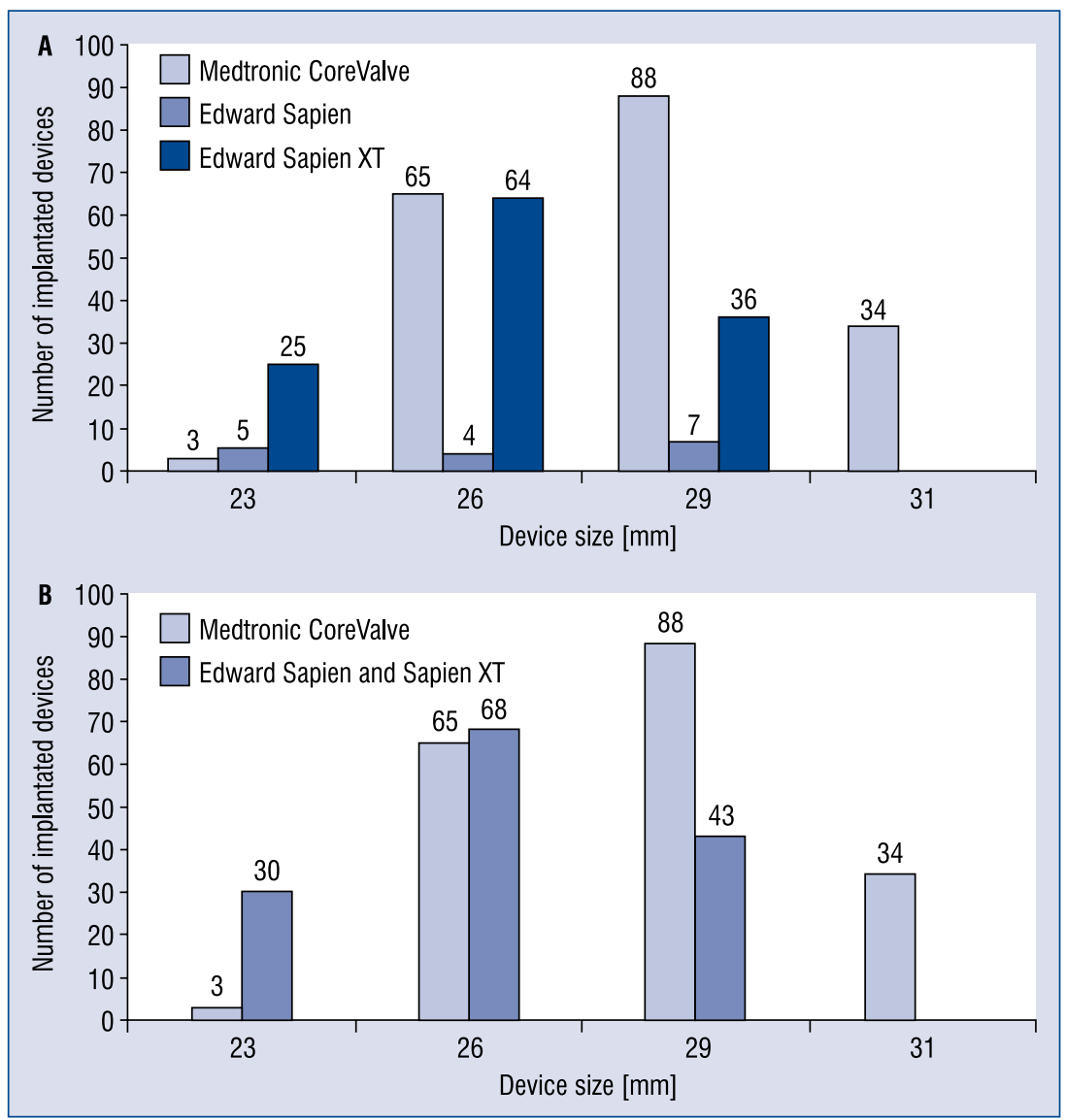

Figure 1. A, B. Aortic valve device size distribution.

symptomatic AS - median aortic valve area was $0.7 \mathrm{~cm}^{2}$ (IQR $0.6-0.8$ ). One hundred and forty-nine (45\%) TAVI procedures were performed in the cathlab. Thirty-two $(9.7 \%)$ procedures were performed in the operating room. One hundred and fifty $(45.5 \%)$ procedures were carried out in the hybrid operating room. Edwards Sapien and Edwards Sapien XT prostheses were implanted in 141 patients (16 and 125, respectively, 42.6\%) and Medtronic CoreValves were implanted in 190 patients $(57.4 \%)$. A detailed distribution of device size is presented on Figure 1A, B. Echocardiography assessment after TAVI procedure revealed that $181(54.7 \%)$ patients developed mild PVL and 22 (6.6\%) patients - moderate PVL. No severe PVLs were observed at those time points. PVL grade remained stable in echocardiography assessment after 6 months of follow-up. Patients with missing echocardiographic assessment of the PVL were excluded from this analysis. Occurrence of moderate PVL was device dependent and more frequent in CoreValve group $(\mathrm{p}=0.02)$. Moreover, PVL after TAVI was observed more often with Edwards
$23 \mathrm{~mm}$ valve and $31 \mathrm{~mm}$ CoreValve prostheses. For the CoreValve devices, bigger size of the implanted device is correlated with larger PVL (Spearman: $\rho=0.19 ; \mathrm{p}=0.01$, Fig. 2 ), but for Edwards Sapien devices this correlation is inverted (Spearman: $\rho=-0.62 ; \mathrm{p}=0.01$, Fig. 3A, B). No significant correlation between PVL and device size was found in the Edwards Sapien XT group (Spearman: $\rho=-0.06$; $\mathrm{p}=0.52$ ) and with whole Edwards (Sapien and Sapien XT) group (Spearman: $\rho=-0.15 ; \mathrm{p}=0.08$ ). Patients with AF had 5 times higher risk of moderate PVL $(p<0.01)$ than patients without AF. Patients treated in hybrid room had 3 times higher risk of moderate PVL than patients treated in the cathlab $(p=0.03)$. Patients treated in the operation room had 6 times higher risk of moderate PVL than patients treated in the cathlab $(\mathrm{p}=0.04)$ (Fig. 4). Median of the procedure time was 135 min (IQR: $105 ; 180)$. Median of the fluoroscopy time was 24 min (IQR: $17 ; 32.875)$. Median of the contrast medium used was $180 \mathrm{~mL}$ (IQR: 140; 210). The highest fluoroscopy time was observed in a group of patients with moderate PVL (Fig. 5). We also 


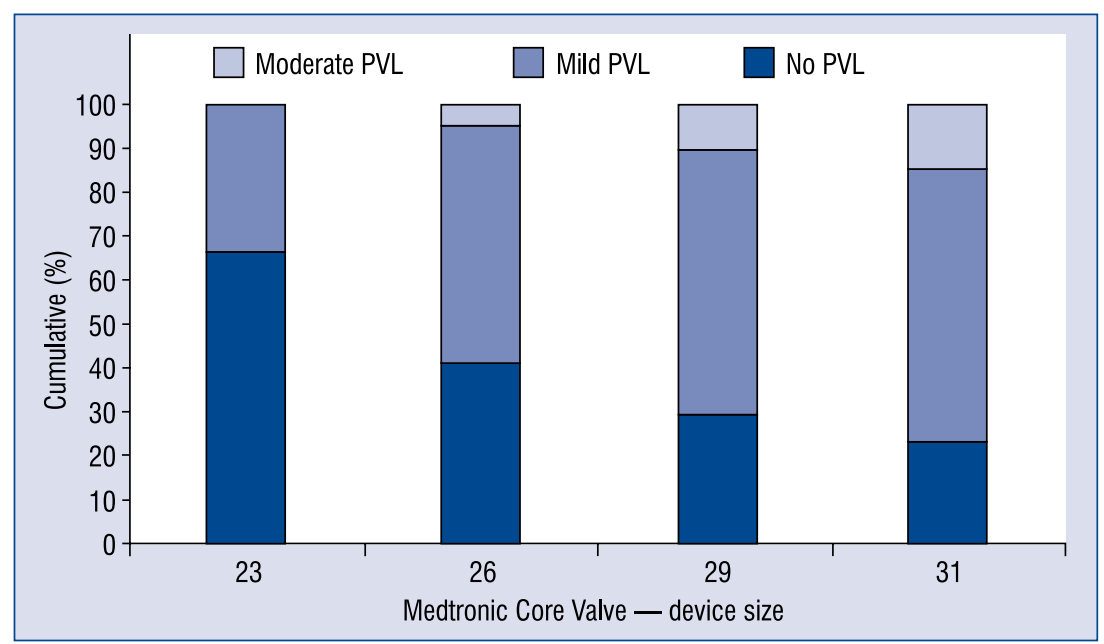

Figure 2. Paravalvular leak (PVL) in a group of CoreValve devices depended on device size.

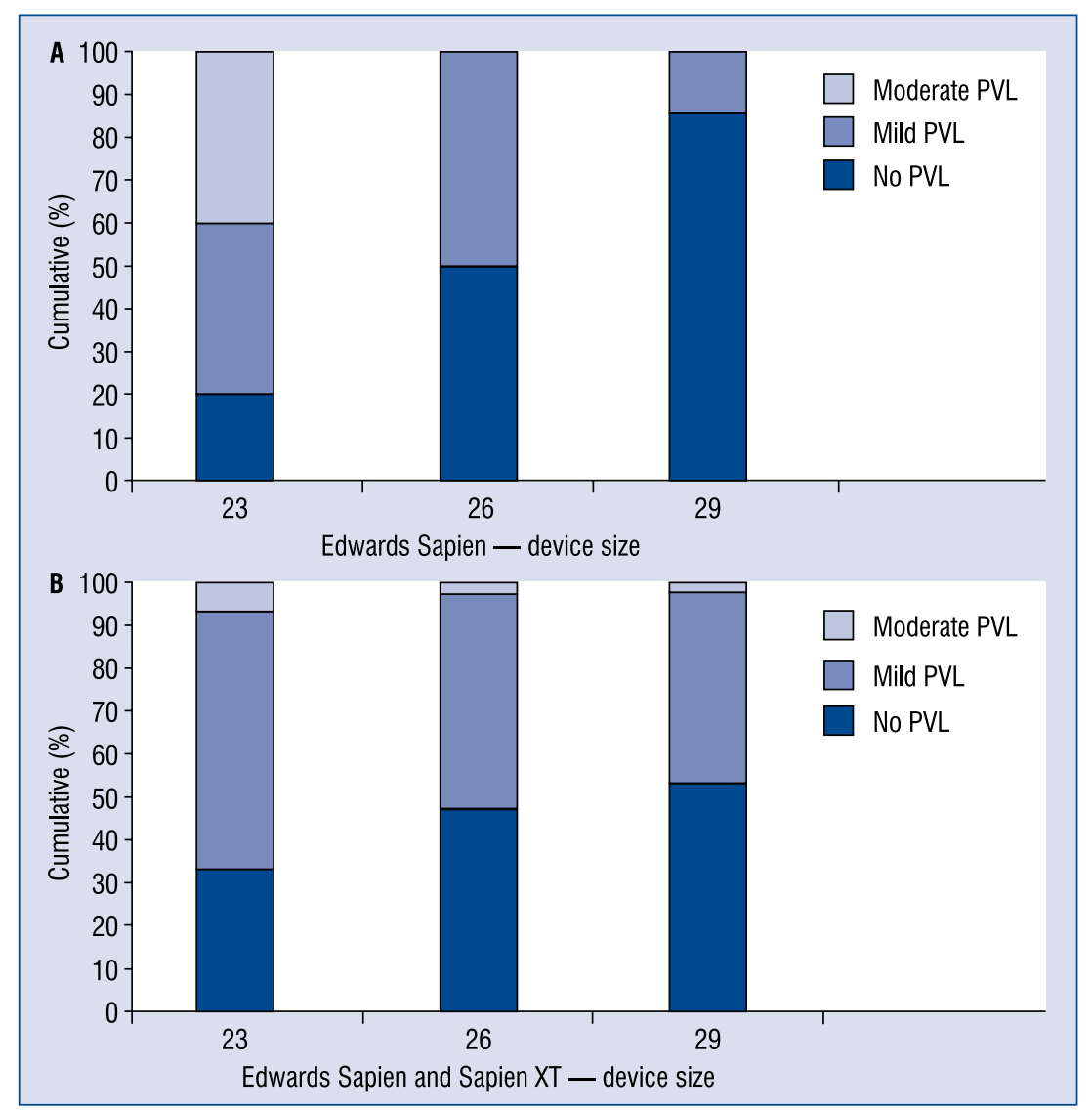

Figure 3. A. Paravalvular leak (PVL) in a group of Edwards Sapien devices depended on device size; B. Paravalvular leak (PVL) in a group of Edwards Sapien and Sapien XT devices depended on device size.

assessed a 6 -month mortality rate that was not significant between patients with no or mild PVL compared to those with moderate PVL ( $p=0.12$, Fig. 6).

\section{Discussion}

TAVI is characterized by a high prevalence of postprocedural PVL, being moderate-to-severe in 


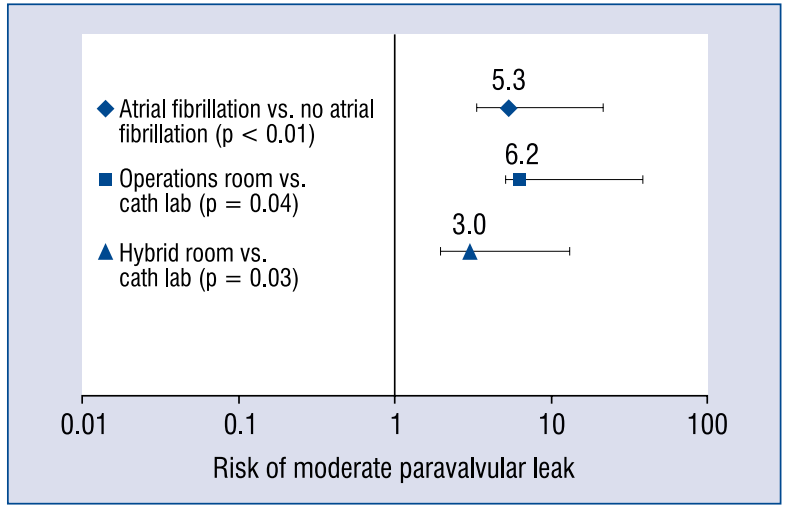

Figure 4. Risk of moderate paravalvular leak occurrence in case of atrial fibrillation and different treatment place (nominal logistic fit).

5-40\% of patients [7-12]. Moderate-to-severe PVL had been previously identified as an independent predictor of late mortality (30 days up to 1 year) after Medtronic CoreValve implantation [9]. An impact of PVL on medium-term prognosis has been recently demonstrated also for the Edwards SAPIEN prosthesis $[12,13]$. Our aim was to assess the incidence of moderate-to-severe PVL after TAVI. Our study examined a cohort of patients from the Polish national POL-TAVI registry, including only patients successfully implanted and discharged alive. According to the registry data, 181 (54.7\%) patients developed mild PVL, 22 (6.6\%) - moderate PVL after 6 months and no severe PVLs were observed. Meta-analyses of multiple studies in the high-risk population show that for either valve type, moderate or severe PVL is an important determinant of mortality $[14,15]$.

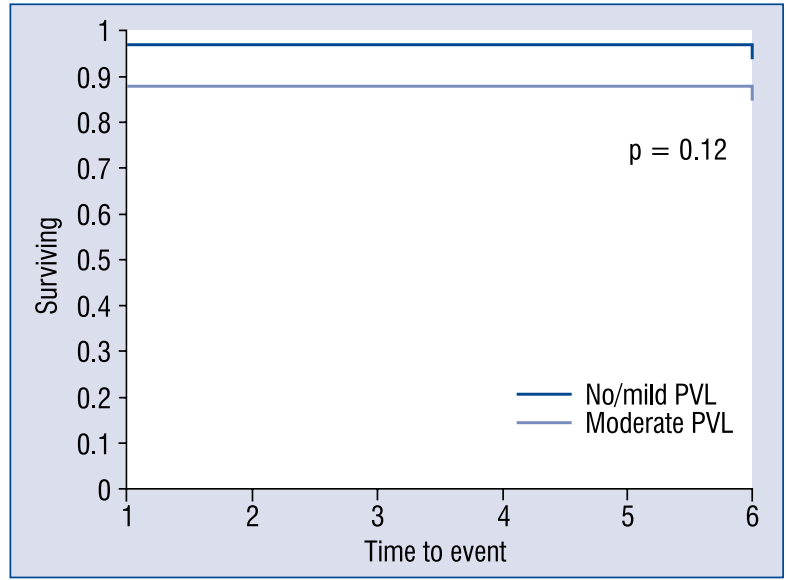

Figure 6. Six-month mortality; PVL — paravalvular leak.

However, in our study, the mortality rate after 6 months was not significant, probably due to low patient count and relatively short period of followup (6 months). Thus, the accurate assessment of this complication is an important means for determining the effectiveness of procedure and should be followed-up in a subset of patients undergoing TAVI. Occurrence of moderate PVL was device dependent and more frequent in the CoreValve group. Moreover, for the CoreValve devices bigger size of implanted device is correlated with larger PVL. This data is accordant with results of other PVL studies [16]. We found an inverted correlation between the degree of PVL and Edwards Sapien device. This could occur due to the possible sizing problem of the device with respect to aortic annulus diameter and learning curve. Operators could eventually downsize the potentially used

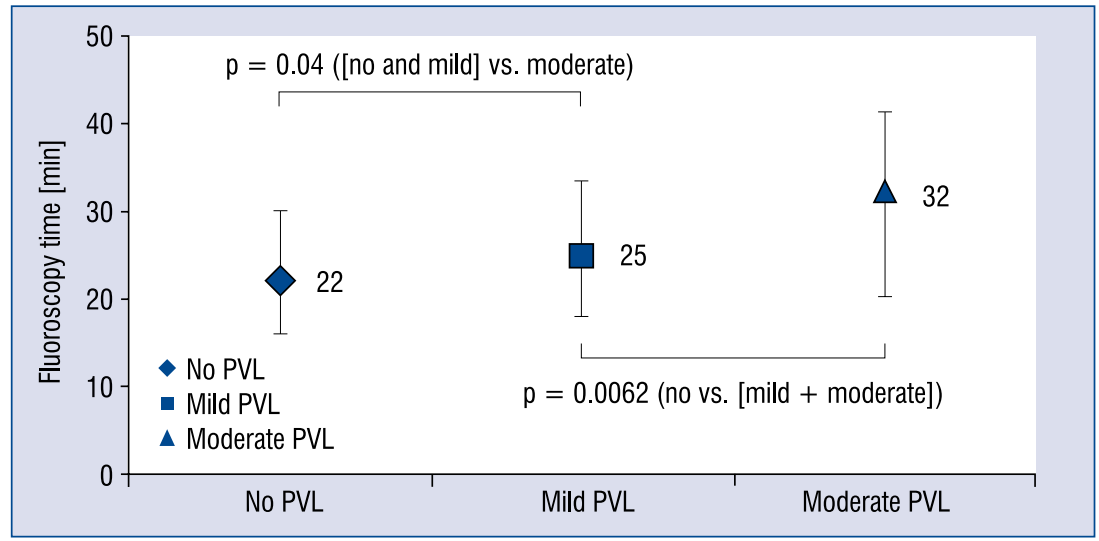

Figure 5. Fluoroscopy time in a group with different paravalvular leak (PVL). 
prosthesis and their decision was affected by the annulus rupture possibility if they chose too big valve. Moreover, we found that at least moderate PVL after TAVI is predicted by preexisting AF and implantation site. There has been no data available so far to support our finding. Differences in PVL occurrence depending on procedure site are probably due to learning curve of the staff and worse quality of imaging in the operating room. Babaliaros et al. [16] compared TAVI via transfemoral approach in the cathlab and the hybrid operating room. Moderate or severe paravalvular leak and device success were similar in the cathlab and hybrid room groups ( $3 \%$ vs. $5.8 \%, \mathrm{p}=0.4 ; 90 \%$ vs. $88 \%, \mathrm{p}=0.79$, respectively) at 30 days.

\section{Limitations of study}

The study involved a relatively small sample size $(\mathrm{n}=331)$ and the data was analyzed retrospectively as registry data. Data was submitted by 20 centers performing TAVI procedures with different grade of completeness. Data submission was not monitored. Correlation of AF and PVL might be a finding by chance due to the number of patients. Most likely root angiography is the preferred method, since echocardiography has its clear limitation in PVL assessment. The precise quantification of the magnitude of PVL still remains a challenging problem.

\section{Conclusions}

TAVI is an efficient treatment for patients with severe AS but may cause a PVL. The occurrence of PVL is associated with device type, size and defined comorbidities. PVL after TAVI was observed more often with Edwards $23 \mathrm{~mm}$ valve and $31 \mathrm{~mm}$ CoreValve prosthesis.

\section{Acknowledgments}

Part of the study was presented as an oral presentation at the $18^{\text {th }}$ International Congress of the Polish Cardiac Society in Poznan, Poland on September $19^{\text {th }}, 2014$.

\section{Conflict of interest: None declared}

\section{References}

1. Leon MB, Smith CR, Mack M et al. Transcatheter aortic-valve implantation for aortic stenosis in patients who cannot undergo surgery. N Engl J Med, 2010; 363: 1597-1607.

2. Kleczyński P, Bagieński M, Sorysz D et al. Short- and intermediate-term mprovement of patient quality of life after transcatheter aortic valve implantation: A single-centre study. Kardiol Pol, 2014; 72: 612-616. doi: 10.5603/KP.a2014.0065.

3. Schewel D, Frerker C, Schewel J et al Clinical impact of paravalvular leaks on biomarkers and survival after transcatheter aortic valve implantation. Catheter Cardiovasc Interv, 2015; 85: 502-514. doi: 10.1002/ccd.25295.

4. Vahanian A, Alfieri O, Andreotti F et al. Guidelines on the management of valvular heart disease (version 2012): The Joint Task Force on the Management of Valvular Heart Disease of the European Society of Cardiology (ESC) and the European Association for Cardio-Thoracic Surgery (EACTS). Eur Heart J, 2012; 33: 2451-2496.

5. Kappetein AP, Head SJ, Généreux P et al. Updated standardized endpoint definitions for transcatheter aortic valve implantation: The Valve Academic Research Consortium-2 consensus document. J Am Coll Cardiol, 2012; 60: 1438-1454. doi: 10.1016/j. jacc.2012.09.001.

6. Détaint D, Lepage L, Himbert D et al. Determinants of significant paravalvular regurgitation after transcatheter aortic valve implantation: Impact of device and annulus discongruence. J Am Coll Cardiol Cardiovasc Interv, 2009; 2: 821-827.

7. Sherif MA, Abdel-Wahab M, Stocker B et al. Anatomic and procedural predictors of paravalvular aortic regurgitation after implantation of the Medtronic CoreValve bioprosthesis. J Am Coll Cardiol, 2010; 56: 1623-1629.

8. Tamburino C, Capodanno D, Ramondo A et al. Incidence and predictors of early and late mortality after transcatheter aortic valve implantation in 663 patients with severe aortic stenosis. Circulation, 2011; 123: 299-308.

9. Takagi K, Latib A, Al-Lamee R et al. Predictors of moderate-tosevere paravalvular aortic regurgitation immediately after CoreValve implantation and the impact of postdilatation. Catheter Cardiovasc Interv, 2011; 78: 432-443.

10. Sinning JM, Hammerstingl C, Vasa-Nicotera M et al. Aortic regurgitation index defines severity of periprosthetic regurgitation and predicts outcome in patients after transcatheter aortic valve implantation. J Am Coll Cardiol, 2012; 59: 1134-1141.

11. Kodali SK, Williams MR, Smith CR et al. Two-year outcomes after transcatheter or surgical aortic valve replacement. N Engl J Med, 2012; 366: 1686-1695.

12. Watanabe $\mathrm{Y}$, Lefèvre $\mathrm{T}$, Arai $\mathrm{T}$ et al. Can we predict post-procedural paravalvular leak after Edwards Sapien transcatheter aortic valve implantation? Catheter Cardiovasc Interv, 2015; 86: 144-151. doi: 10.1002/ccd.25665.

13. Athappan G, Patvardhan E, Tuzcu EM et al. Incidence, predictors, and outcomes of aortic regurgitation after transcatheter aortic valve replacement: Meta-analysis and systematic review of literature. J Am Coll Cardiol, 2013; 61: 1585-1595.

14. O'Sullivan KE, Gough A, Segurado R et al. Is valve choice a significant determinant of paravalular leak posttranscatheter aortic valve implantation? A systematic review and meta-analysis. Eur J Cardiothorac Surg, 2014; 45: 826-833.

15. De Carlo M, Giannini C, Fiorina $\mathrm{C}$ et al. Paravalvular leak after CoreValve implantation in the Italian Registry: Predictors and impact on clinical outcome. Int J Cardiol, 2013; 168: 5088-5089. doi: 10.1016/j.jicard.2013.07.184.

16. Babaliaros V, Devireddy C, Lerakis S et al. Comparison of transfemoral transcatheter aortic valve replacement performed in the catheterization laboratory (minimalist approach) versus hybrid operating room (standard approach): Outcomes and cost analysis. J Am Coll Cardiol Cardiovasc Interv, 2014; 7: 898-904. doi: 10.1016/j.jcin.2014.04.005. 